\title{
Effect of Number of Input Signals on Chaos Synchronization by Applying Zero-dispersion Nonlinear Resonance
}

\author{
Ming-Chi Lu, ${ }^{1}$ Feng-Wei Tsai, ${ }^{1}$ Chen-An Chan, ${ }^{1}$ Yan-Lin Zhong, ${ }^{1}$ \\ Chia-Ju Liu, ${ }^{2}$ Tsung-Lun Tsai, ${ }^{2}$ Jing-Yuan Ko, ${ }^{1}$ and Ming-Chung Ho ${ }^{1 *}$ \\ ${ }^{1}$ Department of Physics, National Kaohsiung Normal University, \\ No. 62, Shenjhong Rd., Yanchao District, Kaohsiung City 824, Taiwan, ROC \\ ${ }^{2}$ Graduate Institute of Science Education \& Environmental Education, National Kaohsiung Normal University, \\ No. 62, Shenjhong Rd., Yanchao District, Kaohsiung City 824, Taiwan, ROC
}

(Received May 30, 2019; accepted December 8, 2020)

Keywords: zero-dispersion nonlinear resonance, phase locking, circuit implementation

In this paper, we report a circuit experiment that demonstrates the effect of the number of input signals on chaos synchronization by applying zero-dispersion nonlinear resonance (ZDNR). The input signals are obtained by generic input-output analysis and the ZDNR theory. In this way, the evolution of chaos synchronization can be observed from our experimental results, that is, two chaotic systems change from phase locking to complete synchronization when the number of input signals of ZDNR increases from one to three. Additionally, a circuit implemented utilizing the ZDNR technique can be applied to sensor circuits to detect specific desired signals.

\section{Introduction}

Chaos is a type of motion that is unique to nonlinear dynamic systems, and is a complex phenomenon that is common in nature. ${ }^{(1,2)}$ How to effectively control chaotic signals to generate valuable applications is a topic that many researchers have studied. ${ }^{(3-7)}$ There are many applications of chaotic synchronization, such as secure communication, ${ }^{(8,9)}$ spread spectrum communication, ${ }^{(10)}$ and information compression and storage. ${ }^{(11)}$ As a result, chaos control and synchronization methods, such as the drive-response method, ${ }^{(12)}$ feedback method, ${ }^{(13)}$ pulse control method, ${ }^{(14)}$ and so forth, ${ }^{(15,16)}$ have been receiving intensive attention and been extensively explored.

In 1994, Soskin proposed an alternative type of nonlinear resonance, ${ }^{(17)}$ called zerodispersion nonlinear resonance (ZDNR). Later, Ho et al. performed a general input-output analysis to verify one of the features of ZDNR, that is, the output response appears without any dispersion or distortion. ${ }^{(18)}$ In 2010 , Ho et al. realized an experimental circuit exhibiting ZDNR. ${ }^{(19)}$ Since then, we have continued to study ZDNR, ${ }^{(20)}$ and extended its application to chaos synchronization. Underlying the mechanism of ZDNR, that is, the output is equal to the input, in this study, we used the general input-output method to calculate the input ZDNR signals as a drive system input into a chaotic system to be output as a response system. When all three input

*Corresponding author: e-mail: t1603@nknucc.nknu.edu.tw https://doi.org/10.18494/SAM.2021.2487 
signals were input, two chaotic systems achieved complete synchronization. However, the type of chaos synchronization changed and phase locking was observed when the number of input ZDNR signals was decreased. The sensitivity of chaos synchronization to ZDNR signals may be applicable to the development of sensor circuits.

\section{Model of Chaos Synchronization by Applying ZDNR}

The following dynamic system is considered:

$$
\ddot{X}+G(\dot{X}, X)+\nabla U=0
$$

where $X$ is the output, $U$ is the potential, and $G$ is used to include the effects of gain and dissipation. Then, we input an external driving force $F$ to the system in Eq. (1) and obtain the following output response system:

$$
\ddot{X}_{1}+G\left(\dot{X}_{1}, X_{1}\right)+\nabla U=F
$$

When the output $X_{1}$ is the same as the external driving force $F$, that is, $X_{1}=F$, the driving input of ZDNR is obtained. Since $X_{1}$ is the same as $F$, we replace $F$ with $X_{1}$ in Eq. (2) to obtain

$$
\ddot{X}_{1}+G\left(\dot{X}_{1}, X_{1}\right)+\nabla U=X_{1}
$$

Taking the system in Eq. (3) as the driving input, the following output response system is obtained:

$$
\ddot{X}_{2}+G\left(\dot{X}_{2}, X_{2}\right)+\nabla U=X_{1}
$$

In this way, $X_{1}$ and $X_{2}$ will eventually achieve chaos synchronization of ZDNR.

\section{Experimental Setup}

A typical Lorenz system is considered as follows:

$$
\left\{\begin{array}{l}
\dot{x}=C_{1}(y-x) \\
\dot{y}=x\left(C_{2}-z\right)-y \\
\dot{z}=x y-C_{3} z
\end{array}\right.
$$

where $\left(C_{1}, C_{2}, C_{3}\right)=(21.3,40.2,3)$. When an external force $F$ is input, the system becomes

$$
\left\{\begin{array}{l}
\dot{x}_{1}=C_{1}\left(y_{1}-x_{1}\right)+F_{1} \\
\dot{y}_{1}=x_{1}\left(C_{2}-z_{1}\right)-y_{1}+F_{2} . \\
\dot{z}_{1}=x_{1} y_{1}-C_{3} z_{1}+F_{3}
\end{array}\right.
$$


By applying generic input-output analysis and ZDNR theory, that is, $\left(F_{1}, F_{2}, F_{3}\right)=\left(x_{1}, y_{1}, z_{1}\right)$, the following driving system is obtained:

$$
\left\{\begin{array}{l}
\dot{x}_{1}=C_{1}\left(y_{1}-x_{1}\right)+x_{1} \\
\dot{y}_{1}=x_{1}\left(C_{2}-z_{1}\right)-y_{1}+y_{1} \\
\dot{z}_{1}=x_{1} y_{1}-C_{3} z_{1}+z_{1}
\end{array}\right.
$$

Then the response output is obtained as

$$
\left\{\begin{array}{l}
\dot{x}_{2}=C_{1}\left(y_{2}-x_{2}\right)+x_{1} \\
\dot{y}_{2}=x_{2}\left(C_{2}-z_{2}\right)-y_{2}+y_{1} \\
\dot{z}_{2}=x_{2} y_{2}-C_{3} z_{2}+z_{1}
\end{array}\right.
$$

We set the scaling factors of the circuit as $\left(x_{1}, y_{1}, z_{1}\right)=\left(10 X_{1}, 10 Y_{1}, 10 Z_{1}\right)$ and $\left(x_{2}, y_{2}, z_{2}\right)=\left(10 X_{2}\right.$, $10 Y_{2}, 10 Z_{2}$ ), and set the time scale as $\tau=1000 t$. Then the driving system in Eq. (7) becomes

$$
\left\{\begin{array}{l}
\dot{X}_{1}=-2130 Y_{1}-2130 X_{1}-100 X_{1} \\
\dot{Y}_{1}=-4020 X_{1}-1000 X_{1} Z_{1}-100 Y_{1}-100 Y_{1} \\
\dot{Z}_{1}=-1000 X_{1} Y_{1}-300 Z_{1}-100 Z_{1}
\end{array}\right.
$$

in which all coefficients must be converted to negative values for the circuit design. Then, the response system in Eq. (8) becomes

$$
\left\{\begin{array}{l}
\dot{X}_{2}=-2130 Y_{2}-2130 X_{2}-100 X_{1} \\
\dot{Y}_{2}=-4020 X_{2}-1000 X_{2} Z_{2}-100 Y_{2}-100 Y_{1} \\
\dot{Z}_{2}=-1000 X_{2} Y_{2}-300 Z_{2}-100 Z_{1}
\end{array}\right.
$$

To transform the above equations into circuits, the systems in Eqs. (9) and (10) are respectively converted to the following systems.

$$
\left\{\begin{array}{l}
\dot{X}_{1}=-\frac{Y_{1}}{R_{3} C_{1}}-\frac{X_{1}}{R_{4} C_{1}}-\frac{X_{1}}{R_{7} C_{1}} \\
\dot{Y}_{1}=-\frac{X_{1}}{R_{10} C_{2}}-\frac{X_{1} Z_{1}}{10 R_{15} C_{2}}-\frac{Y_{1}}{R_{11} C_{2}}-\frac{Y_{1}}{R_{14} C_{2}} \\
\dot{Z}_{1}=-\frac{X_{1} Y_{1}}{10 R_{19} C_{3}}-\frac{Z_{1}}{R_{20} C_{3}}-\frac{Z_{1}}{R_{18} C_{3}}
\end{array}\right.
$$




$$
\left\{\begin{array}{l}
\dot{X}_{2}=-\frac{Y_{2}}{R_{23} C_{4}}-\frac{X_{2}}{R_{24} C_{4}}-\frac{X_{1}}{R_{27} C_{4}} \\
\dot{Y}_{2}=-\frac{X_{2}}{R_{30} C_{5}}-\frac{X_{2} Z_{2}}{10 R_{31} C_{5}}-\frac{Y_{2}}{R_{35} C_{5}}-\frac{Y_{1}}{R_{34} C_{5}} \\
\dot{Z}_{2}=-\frac{X_{2} Y_{2}}{10 R_{39} C_{6}}-\frac{Z_{2}}{R_{40} C_{6}}-\frac{Z_{1}}{R_{38} C_{6}}
\end{array}\right.
$$

In this way, the input and output systems will achieve complete synchronization. An analog circuit simulation of ZDNR on Multisim is shown in Fig. 1, in which the switches control

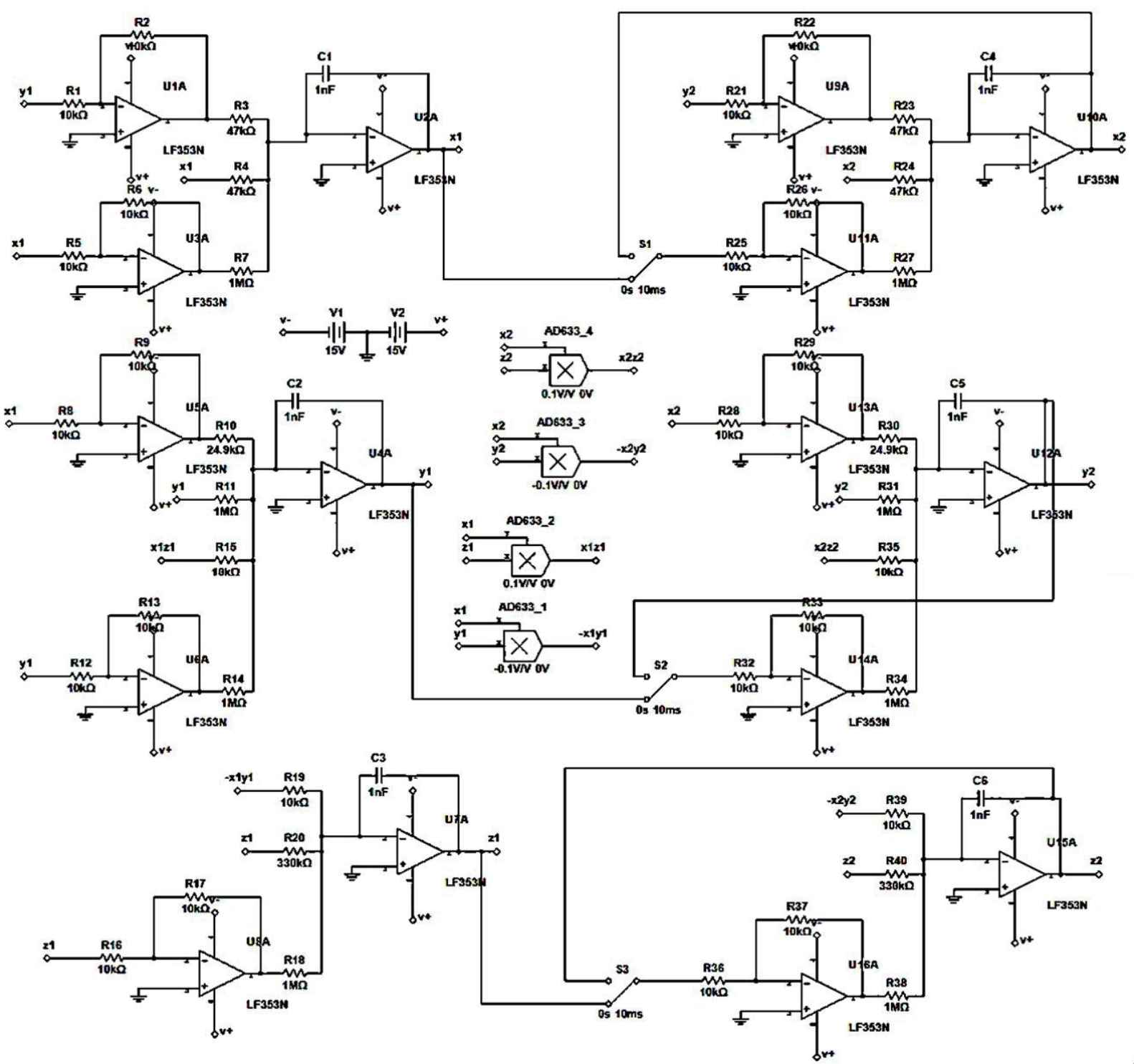

Fig. 1. Circuit diagram of chaos synchronization by applying ZDNR, where S1, S2, and S3 are switches controlling the input signals of ZDNR. 
whether or not driving signals of ZDNR are input by switching ON or OFF, as shown in Fig. 2. Figure 3 shows the setup and the result of implementing the ZDNR phenomenon in a real circuit.

\section{Results}

First, we set all three switches to OFF to ensure that no ZDNR signals are input, and the phase diagrams of the ZDNR signals vs the output signals are measured. From Fig. 4, the phase diagrams clearly exhibit a chaotic state, which means that the output signals and ZDNR signals do not synchronize when no ZDNR signals are input. On the other hand, when we set all three

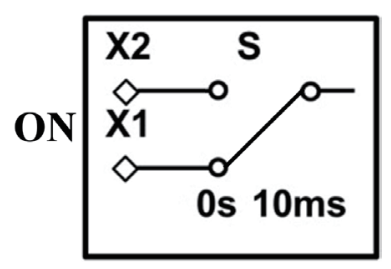

(a)

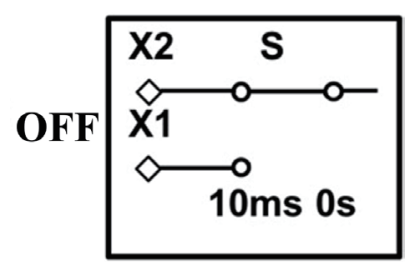

(b)

Fig. 2. Switches. (a) ON corresponds to the input of the ZDNR signal and (b) OFF corresponds to no input of the ZDNR signal.

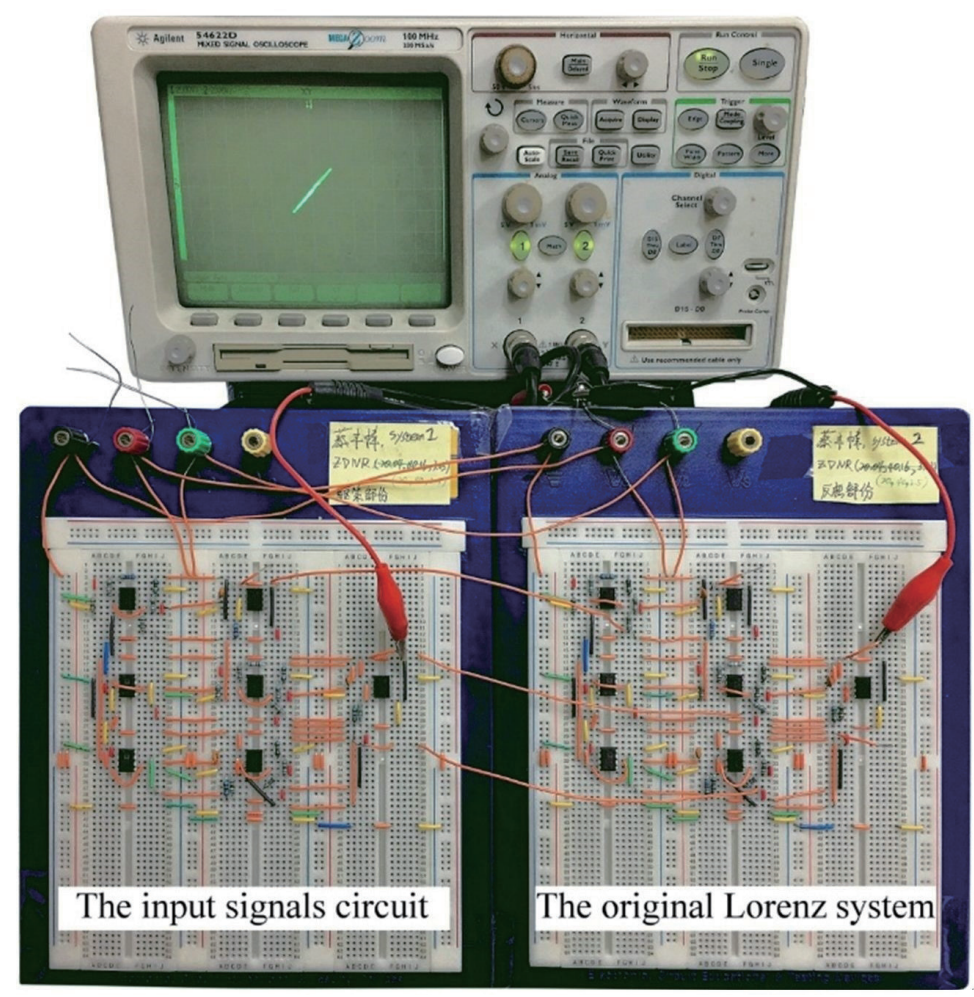

Fig. 3. (Color online) Implementation of ZDNR in real circuit and result obtained from oscilloscope. 


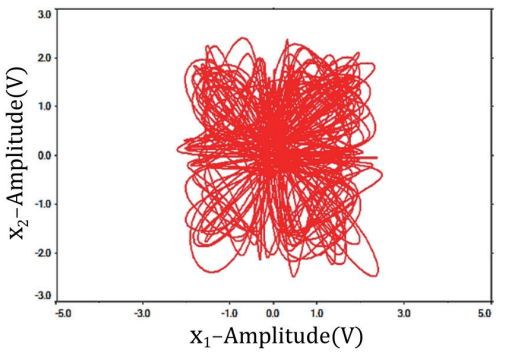

(a)

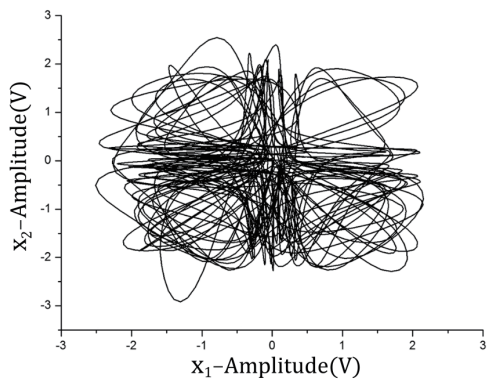

(d)

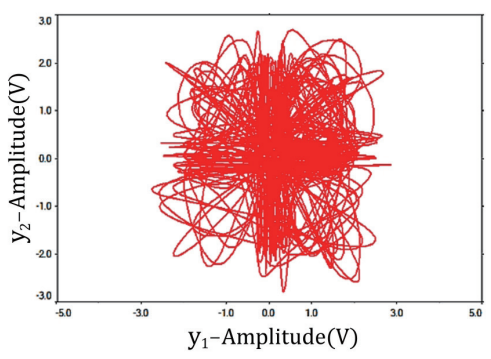

(b)

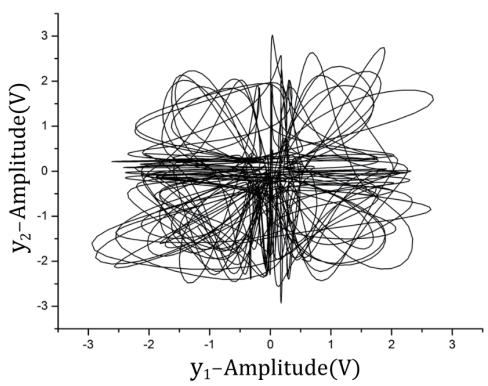

(e)

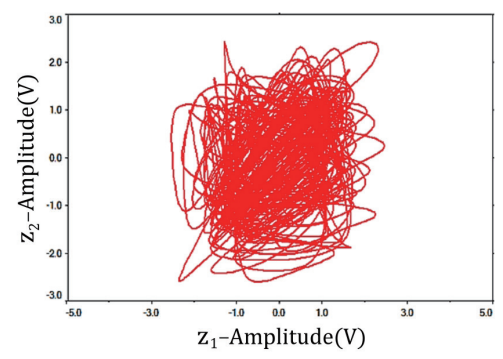

(c)

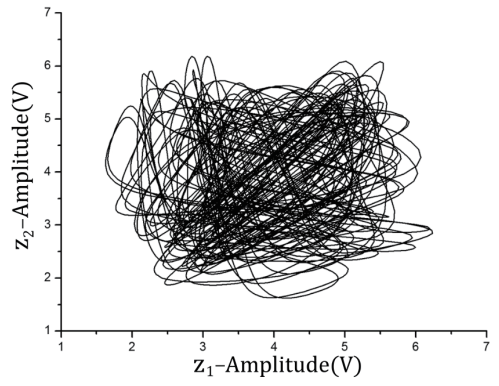

(f)

Fig. 4. (Color online) Phase diagrams of the ZDNR signals $\left(x_{1}, y_{1}, z_{1}\right)$ vs the output signals $\left(x_{2}, y_{2}, z_{2}\right)$ when no ZDNR signals are input. (a), (b), and (c) Results of the simulation. (d), (e), and (f) Results of the real circuit.

switches to ON so that all three ZDNR signals are input, the output signal and the input signal achieve complete synchronization. The phase diagrams of the input signals vs the output signals, as shown in Fig. 5, show diagonal lines, which mean that the input signals and the output signals are identical, that is, they are synchronized.

\section{Discussion}

Since the input of the ZDNR contained three signals, we were interested in the effect of the number of input ZDNR signals on chaos synchronization. Thus, we controlled the switches to change the number of input ZDNR signals from one to three. From the experimental results of the real circuit, the phase diagrams of the input signals vs the output signals displayed a proportional trend when one ZDNR signal was input, as shown in Fig. 6(a). This means that there was a phase-locking relationship between the input and output. The thick diagonal lines shown in Fig. 6(b) mean that there was a quasi-periodic relationship between the input and output when two ZDNR signals were input. The diagonal line shown in Fig. 6(c) means that the input and output achieved complete synchronization when three ZDNR signals were input.

Additionally, to verify the effect of the number of input ZDNR signals on chaos synchronization, we plotted the time series of the output signal, and marked with thick red dots places where the output signal and the input signal are identical. When no ZDNR signal was 


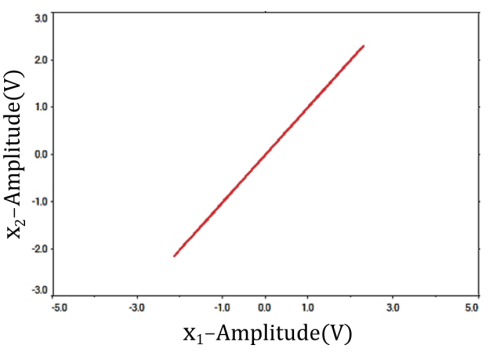

(a)

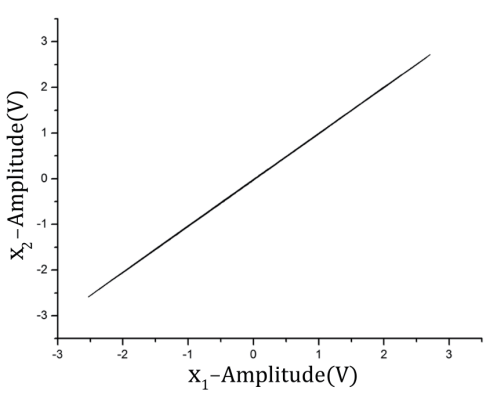

(d)

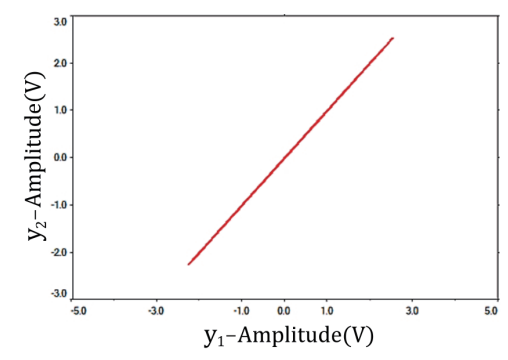

(b)

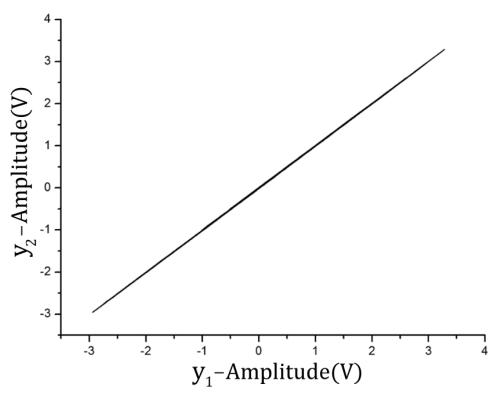

(e)

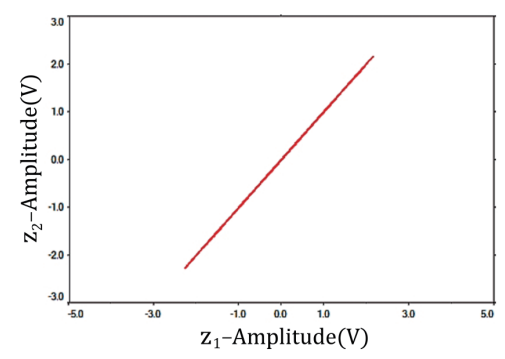

(c)

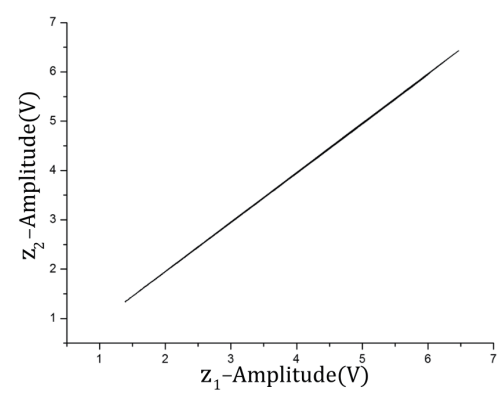

(f)

Fig. 5. (Color online) Phase diagrams of the input signals of ZDNR $\left(x_{1}, y_{1}, z_{1}\right)$ vs the output signals $\left(x_{2}, y_{2}, z_{2}\right)$ when all three ZDNR signals are input. (a), (b), and (c) Results of the simulation. (d), (e), and (f) Results of the real circuit.

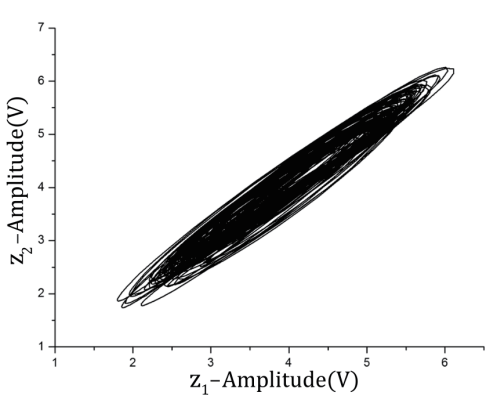

(a)

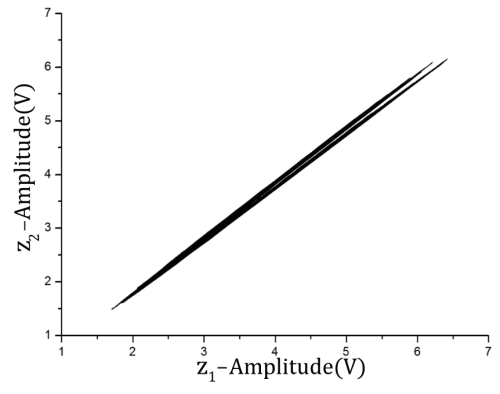

(b)

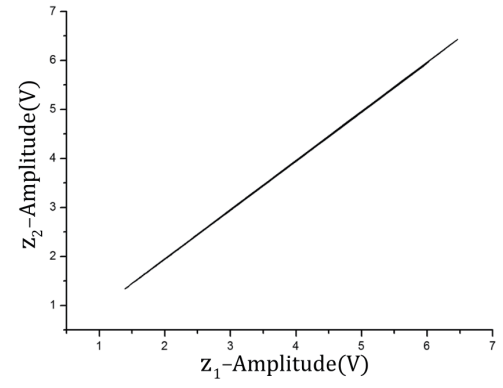

(c)

Fig. 6. Phase diagrams of the input signals of ZDNR $\left(x_{1}, y_{1}, z_{1}\right)$ vs the output signals $\left(x_{2}, y_{2}, z_{2}\right)$ when (a) one of the ZDNR signals is input, (b) two of the ZDNR signals are input, and (c) all three ZDNR signals are input.

input, very few and scattered thick red dots were observed as shown in Fig. 7(a). However, the number of thick red dots increased slightly and the dots became more concentrated when one of the ZDNR signals was input as shown in Fig. 7(b). When two ZDNR signals were input, there were many clusters of thick red dots [Fig. 7(c)]. Finally, when all three ZDNR signals were input, the entire time series of the output was marked with thick red dots as shown in Fig. 7(d), that is, the input and output signals achieved complete synchronization. 


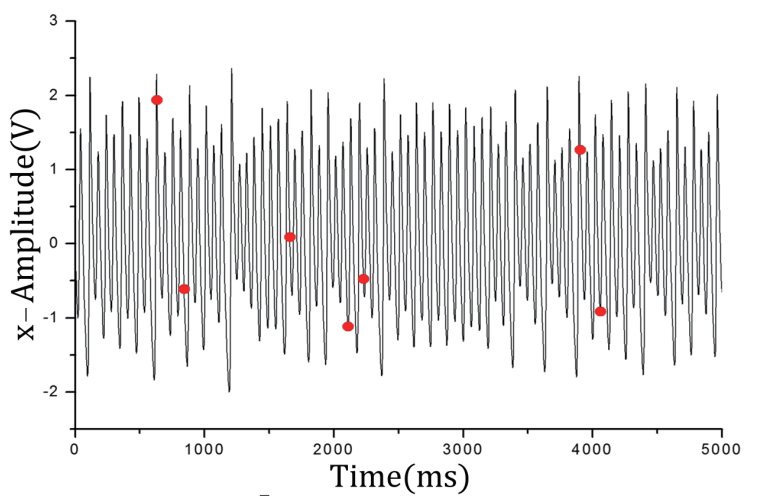

(a)

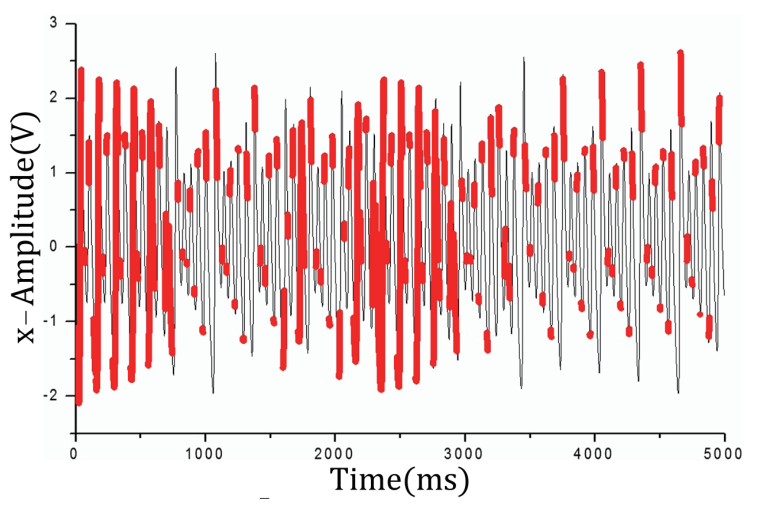

(c)

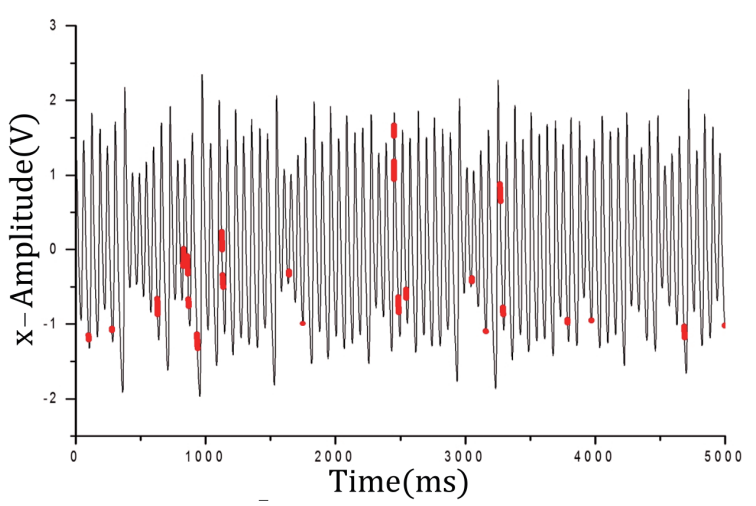

(b)

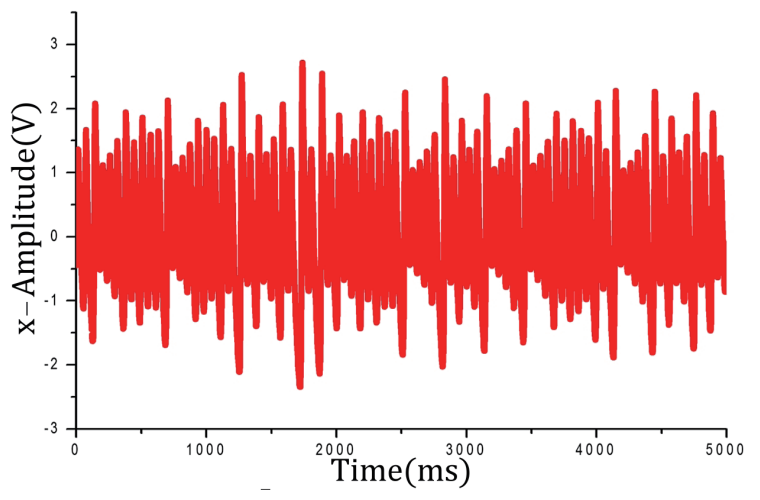

(d)

Fig. 7. (Color online) Time series of the output signals with thick red dots indicating where the input and output are identical. (a) No ZDNR signals are input. (b) One ZDNR signal is input. (c) Two ZDNR signals are input. (d) All three ZDNR signals are input.

\section{Conclusions}

Novel control methods of chaos synchronization have always been interesting research topics. After studying ZDNR and implementing a ZDNR circuit successfully, we extended the application of ZDNR to chaos synchronization. Underlying the mechanism of ZDNR, that is, the output is the same as the input, we used the general input-output method to calculate the ZDNR signals as the input. Finally, we successfully implemented the chaos synchronization circuit of ZDNR. Furthermore, by changing the number of input ZDNR signals, we observed the evolution of chaos synchronization through phase locking, quasi-periodic behavior, and complete synchronization from the experimental results. Furthermore, the sensitivity of circuits to ZDNR signals will be helpful for the design of sensor circuits.

\section{References}

1 E. Mosekilde, J. Aracil, and P. M. Allen: Syst. Dyn. Rev. 4 (1998) 14. https://doi.org/10.1002/sdr.4260040104

2 A. Kaplan and J. K. Garner: Dev. Psychol. 53 (2017) 2036. https://doi.org/10.1037/dev0000339 
3 S. Boccaletti, C. Grebogi, Y. C. Lai, H. Mancini, and D. Maza: Phys. Rep. 329 (2000) 103. https://doi. org/10.1016/S0370-1573(99)00096-4

4 E. Ott and M. Spano: AIP Conf. Proc. 375 (1996) 92. https://doi.org/10.1063/1.51017

5 L. W. Pecora and T. L. Carroll: Phys. Rev. Lett. 64 (1990) 821. https://doi.org/10.1103/PhysRevLett.64.821

6 U. Dressler and G. Nitsche: Phys. Rev. Lett. 68 (1992) 1. https://doi.org/10.1103/PhysRevLett.68.1

7 X. Chai, X. Fu, Z. Gan, Y. Zhang, Y. Lu, and Y. Chen: Neural Comput. Appl. 32 (2020) 4961. https://doi. org/10.1007/s00521-018-3913-3

8 T. Yang and L.O. Chua: IEEE Trans. Circuits Syst. I Fundam. Theory Appl. 43 (1996) 817. https://doi. org/10.1109/81.536758

9 F. Moez: Chaos, Solitons Fractals 18 (2003) 141. https://doi.org/10.1016/S0960-0779(02)00585-4

10 G. Heidari-Bateni and C. D. McGillem: IEEE Trans. Commun. 42 (1994) 1524. https://doi.org/10.1109/ TCOMM.1994.582834

11 B. Hahn: Commun. ACM 17 (1974) 434. https://doi.org/10.1145/361082.361086

12 M. Hu, Z. Xu, and Y. Yang: Physica A 387 (2008) 3759. https://doi.org/10.1016/j.physa.2008.02.066

13 J. A. Hołyst and K. Urbanowicz: Physica A 287 (2000) 587. https://doi.org/10.1016/S0378-4371(00)00395-2

14 S. F. Masri, G. A. Bekey, and T. K. Caughey: J. Appl. Mech. 48 (1981) 619. https://doi.org/10.1115/1.3157684

15 S. Chen, Q. Zhang, J. Xie, and C. Wang: Chaos, Solitons Fractals 20 (2004) 947. https://doi.org/10.1016/ j.chaos.2003.09.021

16 H. Li, X. Liao, C. Li, and C. Li: Neurocomputing 74 (2011) 3212. https://doi.org/10.1016/j.neucom.2011.05.002

17 S. M. Soskin: Phys. Rev. E 50 (1994) R44. https://doi.org/10.1103/PhysRevE.50.R44

18 M. C. Ho, J. Y. Ko, T. H. Yang, and J. L. Chern: Europhys. Lett. 48 (1999) 603. https://doi.org/10.1209/epl/ i1999-00526-5

19 M. C. Ho, C. H. Lin, M. C. Lu, T. F. Hsu, C. J. Liu, C. T. Kuo, and I. M. Jiang: Phys. Lett. A 374 (2010) 2624. https://doi.org/doi:10.1016/j.physleta.2010.04.043

20 M. C. Ho, H. Jan, C. J. Liu, T. F. Hsu, I. M. Jiang, S. Bu, and M. C. Lu: Chin. J. Phys. 48 (2010) 535. http://www. airitilibrary.com/Publication/alDetailedMesh?DocID=05779073-201010-201011300178-201011300178-535-545 